\title{
Italique
}

Poésie italienne de la Renaissance

XVI | 2013

Varia

\section{Paralipomeni della disputa Bembo-Brocardo}

\section{Danilo Romei}

\section{(2) OpenEdition \\ Journals}

Edizione digitale

URL: http://journals.openedition.org/italique/368

DOI: 10.4000/italique.368

ISSN: 1663-4438

\section{Editore}

Librairie Droz

\section{Edizione cartacea}

Data di pubblicazione: 1 dicembre 2013

Paginazione: 69-77

ISBN: 978-2-600-01779-4

ISSN: 1423-3983

Notizia bibliografica digitale

Danilo Romei, « Paralipomeni della disputa Bembo-Brocardo », Italique [Online], XVI | 2013, online dal 01 décembre 2016, consultato il 20 avril 2019. URL : http://journals.openedition.org/italique/368

DOI : 10.4000/italique.368 


\section{Danilo Romei}

PARALIPOMENI DELLA DIS P UTA B E M B O - B R O C A R D O 



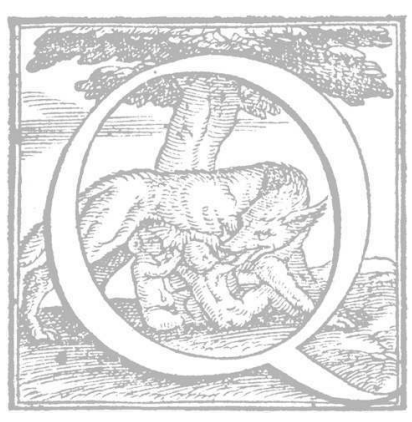

ualche anno fa mi capitò di editare una serie di sonetti vituperosi pertinenti alla "disputa Bembo-Brocardo" compresi nel Cod. Marc. It. XI 66 (=6730). ${ }^{\mathrm{I}} \mathrm{Di}$ recente, scorrendo la versione elettronica della Rime del Brocardo, della quale la Biblioteca Italiana (BIT) attribuisce le cure a Caterina] Saletti con la data del 2005, senza fare alcun riferimento a fonti o edizioni cartacee, ${ }^{2}$ mi è saltato agli occhi, proprio in coda al testo, con il numero d'ordine 47 , il sonetto che trascrivo con qualche minimo ritocco (per uniformare lo stile delle citazioni):

Dio capra e uomo e lana e corne, e voi, nove intatte sorelle oneste e dive, che mai di grazie vi mostrasti schive, deh, venitene a star un po' con noi!

Vi chiama Alcippo, Alcippo che, di poi ch'a bocca poste s'ha le vostre pive, risuonan tute l'antenoree rive, e Titiro si sta a pascer boi.

Datemi un sì bel stil e tanta lena ch'io possi abbatter qui questa puttana e cacciarti nel cul quel ziccolone.

Io dico a te, di lodovica vena, d'amaro ragionar, lingua roffiana, e cerre e seste zucca di melone.

Non c'è alcun dubbio che il sonetto appartenga alla fiorita sequela di oltraggi poetici che i belligeranti della querelle si scambiarono nel corso del I53I. Qualche problema solleva l'attribuzione al Brocardo, che l'assetto testuale della BIT sembra dare per scontato, senza peraltroripeto - indicare la fonte del testo. Il quale non compare nella stampa "canonica" (ed unica) delle rime del Brocardo, ${ }^{3}$ né in quella edizioncina che Domenico Vitaliani allesti nel 1902 in appendice alla sua monografia brocardiana. ${ }^{4}$

Ma sarà meglio (a beneficio del lettore e mio) procedere con ordine, riassumendo $i$ dati principali della questione quali si sono delineati 
nella mia precedente ricognizione dei testi. Una disputa BemboBrocardo non è mai esistita, nel senso che i due presunti protagonistiche si sappia - non si sono mai affrontati direttamente. Sulla base dei documenti noti, sembra che il putiferio sia nato da un sonetto pastorale che Bernardo Tasso inseri nel suo primo libro degli Amori, che converrà rileggere.

Agreste iddio, a cui più tempi alzaro

i pastori d'Arcadia, ov'ancor vive

il tuo nome onorato, e ne le olive

scritto e negli olmi a' quai sotto cantaro,

questa sampogna, al cui soave e chiaro

suono talora a le dolc[i] ombre estive

cantar solea ne l'antenoree rive

Titiro, fra' pastor famoso e raro,

vinse Alcippo cantando; e a te la dona, appendendola lieto a questo faggio, d'odorati e bei fior cinto la fronte;

e grida: - O Pan, o Pan, sempre sien pronte

le mie voci in lodarti; e al novo maggio

le corna t'ornerà verde corona. ${ }^{5}$

Era pacifica l'identificazione di Alcippo con il Brocardo; $i$ lettori della parte ostile ravvisarono in Titiro, "famoso e raro», ma sconfitto nella gara poetica, l'illustre Pietro Bembo, che in effetti si era attribuito come tanti altri, tuttavia - lo pseudonimo virgiliano: di qui la denuncia di sconsiderata impudenza a carico del Tasso e del Brocardo. Questi si affrettarono a smentire l'interpretazione, ma ormai il danno era fatto. Si dice che il Bembo medesimo se ne risentisse e invocasse l'intervento vendicatore di un professionista del vituperio qual era Pietro Aretino. Chissà se è vero. In ogni caso l'A retino rovesciò sui malcapitati uno o più sonetti obbrobriosi (probabilmente quattro, identificabili per congettura all'interno del corpus marciano), ai quali la parte avversa non mancò di rispondere a tono. La faccenda andò avanti per qualche mese nell'estate del I53I, fino alla nota conclusione: il Brocardo venne meno fra il 27 e il 28 agosto I53I. ${ }^{6}$ Giovanni Brevio attribui la causa della morte al "fastidio postosi de li sonetti scrittili contra». ${ }^{7}$ L'Aretino, pur componendo altri quattro sonetti di onoranza funebre, non mancò di menare vanto di aver ammazzato il Brocardo con $i$ suoi versi. 
Il sonetto della BIT risulta con evidenza una risposta (ma non per le rime) al primo dei sonetti del corpus marciano, di pertinenza aretiniana assai probabile, che qui trascrivo.

Dio capra ed om, dal culo in giù di lana, dal culo in sù robba pilosa e schiva, ti sacro questa bella e nuova piva, che non bee vino e pare una campana, con cui Alcippo l'altra setimana, qual sa la furfantesca comitiva, Titiro vinse a l'ombra d'una oliva a cantar bestialmente una pavana.

Prèndila adon(que) e p(er)ché lei s'adori chiama ogni gentil bue dil novo armento a farle intorno una osteria di fiori;

e se gli avvien che 'l novo eroico vento facia il corpo gonfiar troppo a' pastori, prèstagliela $\mathrm{p}(\mathrm{er})$ calza di argomento.

Basterebbe il ricalco quasi perfetto del primo emistichio dell'incipit (Dio capra ed om / Dio capra e uomo) a dimostrarlo, con la convocazione parallela dei protagonisti Alcippo/Titiro nella seconda quartina; ma si può portare a ulteriore conferma la ritorsione del motivo della piva/pive e del bue/boi. In piu il sonetto di parte brocardiana sembra ritornare in qualche modo alla scaturigine della querelle, riesumando le «antenoree rive» (ovvero le rive del Brenta o del Bacchiglione, che costeggiano la città fondata da Antenore) che già comparivano - nella stessa posizione - nel sonetto tassiano degli Amori (v. 7).

Circa la partenità brocardiana del sonetto, gioverà ricordare che fin dal nascere della questione il Tasso e il Brocardo rivelano temperamenti e impulsi diversi se non opposti. Mentre il primo mostra subito una propensione alla prudenza e alla diplomazia e anzi dichiara apertamente la sua avversione a esporsi in scontri spericolati, cercando di sopire e di parlamentare (fin troppo sommessamente), il secondo, al contrario, si mostra cosi «alterato» per l'oltraggiosa provocazione da far temere che possa trascendere a fare «cosa indegna di lui»». ${ }^{8}$ Che qualche cosa facesse è probabile. Si dovranno almeno ricordare $i$ due «oscurissimi〉 sonetti gergali in vituperio di Pietro Aretino La ludovica calca, 
vil baccone $e$ Pietro Aretin, che la tua serpentina, trascritti $d a$ Rodolfo Renier dal cod. Campori 425 della Biblioteca Estense. ${ }^{9}$ La loro pertinenza al contesto della polemica è tutt'altro che certa, anche per le oggettive difficoltà esegetiche che imbrogliano la lettura, tuttavia non si potrà non rimarcare la coincidenza dell'epiteto gergale ludovica/lodovica tanto nell'incipit di uno di questi sonetti quanto al $v$. I2 del sonetto di cui si fa discorso.

Ma si può dire di più. Si può citare un documento che sembra attagliarsi esattamente alla circostanza di questo sonetto. Si tratta di una lettera di Bernardo Tasso datata "Di Padova, il giorno XXI di Luglio del XXXID, e indirizzata nientemeno che Al Molto Mag. ${ }^{\text {co }}$ Signor il Signor Pietro Aretino Mio Onorato:

Signor Pietro mio onorato, non vorrei che vi credesti che tenesse sì poca memoria di voi, che non avesse fatto quello uffizio qui ch'io devevo prima, con li Signori Abbati Cornari, li quali si dogliono e dolsero meco che avesti tale opinione di loro, e mi risposero liberamente pregandomi ch'io vi scrivesse che non aveano detto tal parole, né le cose vostre meritano che le potessero dire, e che chi le aveva o detto o scritteli mentiva per la gola, et era persona di mala natura, e faceva mal uffizio. E trovarono il Querini, e si dolsero seco in colera. Il quale loro rispose con mille giuramenti che non ne sapea cosa alcuna, e che non ve lo avea né detto né scritto. Il Brocardo similmente, e vi rende certo di non aver fatto tal giudizio, né esser per farlo; anzi dove potrà è disposto a farvi apiacere. Dil Sonetto, al mio ritorno qui avevano ritrovato l'autore e di già rispostoli. Doppoi se ne sono fatti tre o quattro, fra li quali ne fo veduto uno in man del Quirini, il titulo del qual era Il Brocardo contra Pietro Aretino, del quale lui non ne vol far altra scusa con voi, per non tenervi di sì poco giudizio che non conosciate se gli è suo. Fra l'altre cose non intende che si voglia dire, e par più tosto sia fatto contra una puttana. Bastivi che tutti sinceramente sono vostri, e io insieme con loro; e quando verranno a Vinezia vi verranno a vedere. Io ritornarò tosto a salutarvi; in questo mezzo tenete memoria di me.

Qualche piccola precisazione non sarà fuori luogo. I «Signori Abbati Cornari» qui menzionati sono tre personaggi della illustre famiglia veneziana dei Cornaro (o Corner), che, insigniti tutt'e tre di una prebenda abbaziale, nel I53I avevano casa in comune a Padova e raccoglievano attorno a sé un circolo culturale nel quale proprio il Tasso 
e il Brocardo avevano un ruolo d'eccellenza. Devono aver avuto una qualche parte nella disputa, ovviamemente non favorevole all'Aretino, che non siamo in grado di precisare.

Ma ciinteressa soprattutto questo sonetto intitolato Il Brocardo contra Pietro Aretino che è in possesso di Luigi Querini (attivo corrispondente padovano dell'Aretino in questo frangente) $e$ che "par [...] sia fatto contra una puttana». Sembra proprio il nostro sonetto, nel quale al v. Io irrompe l'epiteto ingiurioso «questa puttana» e che ha nelle terzine uno sviluppo alquanto oscuro. Che il Tasso metta in dubbio l'orientamento antiaretiniano di questi versi, valendosi pretestuosamente delle ambiguità del testo, rientra nella sua politica di prudente mascheramento e non c'è da credergli più del ragionevole. Il disconoscimento della paternità del testo da parte del Brocardo (ammesso che il Tasso dica il vero) sarà da registrare con beneficio d'inventario. E tutt'altro che da escludere che chi ha tirato il sasso non nasconda la mano.

Vorrei poter sciogliere $i$ nodi esegetici che pongono le terzine, ma non sono da tanto. E probabile il ricorso di elementi di quella lingua furbesca, ovvero di quel gergo (forse piuttosto goliardico che autenticamente malavitoso) che godeva di tanta fortuna nella Padova universitaria di quegli anni. Non ho mancato di consultare il Nuovo modo de intendere la lingua zerga, ${ }^{10}$ che si suole attribuire al Brocardo medesimo, nonché gli studi e $i$ dizionari specializzati, ma non sono approdato a niente di persuasivo e preferisco evitare le ipotesi campate in aria. Non si può escludere neppure qualche guasto testuale; in effetti la correttezza sintattica richiederebbe, al posto del cacciarti del v. II, un più conseguente cacciarli; ma l'intervento rischia di essere banalizzante. Ribadisco, in ogni caso, che a mio parere la «puttana» e la «lingua roffiana» delle terzine hanno a bersaglio Pietro Aretino.

Danilo Romei 


\section{Danilo Romei}

I. Danilo Romer, Pietro Aretino tra Bembo e Brocardo (e Bernardo Tasso), in Studi sul Rinascimento italiano / Italian Renaissance Studies. In memoria di Giovanni Aquilecchia, a cura di Angelo Romano e Paolo Procaccioli, Manziana, Vecchiarelli Editore, 2005 («Cinquecento», Testi e Studi di Letteratura Italiana / Studi, I 5), pp. I43-I6r. A questo saggio rimando sia per il complesso delle informazioni (che sarebbe troppo lungo e sicuramente inutile riproporre) sia per la bibliografia pregressa.

2. A dire il vero, in questo momento, all'indirizzo telematico della BIT compare soltanto il messaggio: «Biblioteca Italiana è in fase di aggiornamento e ristrutturazione. La nuova versione del sito, modificata nell'architettura di insieme e con un consistente incremento di dati, sarà consultabile a partire dal marzo 20 I 2».

3. Rime del Brocardo et d'altri authori [raccolte da Francesco Amadi]. Colophon: Stampate in Venetia, I538. Il mese di decembre.

4. Antonio Brocardo: una vittima del bembismo, Lonigo, Tip. Papolo \& Granconato, 1902. Non sono mai riuscito a vedere la monografia della quale ho trovato soltanto questa sommaria indicazione bibliografica: C. MiAni, Una contesa letteraria del Cinquecento: Bembo e Brocardo, Sassari 1904 (non compare neppure nell'OPAC dell'SBN).

5. Cito dall'editio princeps, Libro primo de gli Amori di Bernardo Tasso. Colophon: In Vinegia per Giouan Antonio \& Fratelli da Sabbio. MDXXXI. Il sonetto è il [ı 26], a c. $48 \mathrm{v}$; il numero d'ordine del componimento (che indico fra parentesi quadre) è conforme a quello della moderna edizione di Chiodo e Martignone: Bernardo TAsso, Rime, vol. I, I tre libri degli Amori, a c. di Carmine Chiodo, e vol. II, Libri Quarto e Quinto. Salmi e Ode, a c. di Vercingetorige Martignone, Torino, Edizioni RES, I995 («Feronia», Collezione di poesia, n. 4). Il motivo della gara poetica tra Titiro ed Alcippo ritorna nei sonetti Pastor, poi s'anicina il chiaro raggio ([I 27], c. 49r) e Se da l'orgolio del gelato nerno ([i 29], c. 49v.

6. Vedi Caterina SaletTi, Un sodalizio poetico: Bernardo Tasso e Antonio Brocardo, in Per Cesare Bozzetti. Studi di letteratura e filologia italiana, a cura di Simone Albonico, Andrea Comboni, Giorgio Panizza, Claudio Vela, Milano, Fondazione Arnoldo e Alberto Mondadori, 1996 («Testi e strumenti di filologia italiana» / Strumenti, 2), pp. 409-24, p. 409.

7. «La morte sua, per giudizio de la maggior parte, è causata dal fastidio postosi de li Sonetti scrittili contra; e io ne posso far fede, perché due giorni prima ch'egli si mettesse al Letto, si sfogò molto meco» (Lettere scritte a Pietro Aretino, a cura di Paolo Procaccioli, I 94, Roma, Salerno Editrice, 2003 [ «Edizione Nazionale delle Opere di Pietro Aretino», vol. Ix, tomo I], pp. Iо8-ıо9).

8. Così si legge in una conciliante missiva (purtroppo senza data) di Bernardo Tasso a Giovan Francesco Valerio (Delle Lettere di M. Bernardo Tasso accresciute, corrette, e illustrate volume primo. Con la Vita dell'Autore scritta dal Sig. AntonFederigo Seghezzi, e con la giunta de' Testimonj più notabili, e degl'Indici copiosissimi. In Padova. (MD)ccxxxiII. Presso Giuseppe Comino, pp. 85-86).

9. Rodolfo Renier, Cenni sull'uso dell'antico gergo furbesco nella letteratura italiana, in Svaghi critici, Bari, Gius. Laterza \& Figli, I910, pp. I-30 (già in Miscellanea di studi 
critici in onore di Arturo Graf, Bergamo, Istituto d'arti grafiche, I908), p. I 8; vedi anche Raimondo Vandini, Appendice dei codici e manoscritti già posseduti dal marchese Campori, Modena, Tip. Domenico Tonietto, i 886, vol. I, p. I 5 I.

Io. La princeps: NVOVO MODO DE / INTENDERE LA LINGVA / ZERGA. Cioe Parlare Forbescho. / Nouellamente posto in luce per ordine / di Alfabeto. OPERA non men / Piaceuole che utilissima. // M. D. XLV. [colophon: In Ferrara per Giouanmaria di Michieli et Antonio Maria di Siuieri compagni. Anno M.D.XLV.] Ho utilizzato il testo trascritto criticamente in Teresa Cappello, Saggio di un'edizione critica del "Nuovo modo de intendere la lingua zerga", in «Studi di filologia italiana», XV (1957), pp. 303-399. 